\title{
Continuous, non-invasive measurement of the haemodynamic response to submaximal exercise in patients with diabetes mellitus: evidence of impaired cardiac reserve and peripheral vascular response
}

\author{
D Joshi, ${ }^{1}$ A Shiwalkar, ${ }^{1}$ M R Cross, ${ }^{2}$ S K Sharma, ${ }^{3}$ A Vachhani, ${ }^{1}$ C Dutt ${ }^{1}$
}

${ }^{1}$ Torrent Research Centre, Village Bhat, Gandhinagar, Gujarat, India; ${ }^{2}$ Veeda Clinical Research, Old Convent of Notre Dame, Derriford, Plymouth, UK; ${ }^{3}$ Veeda Clinical Research, Ambawadi Ahmedabad, Gujarat, India

Correspondence to:

Dr Chaitanya Dutt, Torrent

Research Center, PO Bhat,

District of Gandhinagar 382428,

Gujarat, India; cdutt@

torrentpharma.com

Accepted 29 September 2009

\section{UNLOCKI}

This paper is freely available online under the BMJ Journals unlocked scheme, see http:// heart.bmj.com/info/unlocked.dtl

\begin{abstract}
Background: Reduced exercise capacity in diabetics has been attributed to limitations in cardiac function and microvascular dysfunction leading to impaired oxygen supply and nutritive perfusion to exercising muscles. Objective: To study changes in cardiac function and microvascular utilisation during exercise in diabetic individuals compared to age-matched controls.
\end{abstract}

Methods: Diabetics with glycosylated haemoglobin $\left(\mathrm{HbA}_{1 \mathrm{c}}\right)<8(\mathrm{n}=31)$, diabetics with $\mathrm{HbA}_{1 \mathrm{c}} \geqslant 8(\mathrm{n}=38)$ and age-matched non-diabetic controls $(n=32)$ performed exercise at $50 \mathrm{~W}$ for 10 minutes followed by recovery, with continuous monitoring of cardiac function by impedance cardiography and regional flow and oxygen saturation by laser Doppler and white light spectroscopy. Results: In the diabetics, cardiac reserve during exercise and cardiac overshoot during recovery are significantly reduced because of reduction in capacity to increase stroke volume. Regional flow to the exercising muscle is reduced and there is also disproportionately greater desaturation of the regional flow. Abnormalities in cardiac function and regional perfusion are related to the severity of diabetes.

Conclusion: Cardiac response to exercise is attenuated significantly in diabetic individuals. Simultaneously, there is impairment in the regional distribution. These changes could be the harbinger of reduced exercise capacity in diabetics.

Clinical studies have shown that limitation in exercise capacity is a strong predictor of cardiovascular and all-cause mortality in diabetic patients with heart failure. Underlying pathophysiology is multifactorial and involves alterations in ventricular-vascular coupling consisting of cardiac changes on the one hand and compromised distribution at the regional microvasculature on the other. If such changes can be detected early (stage A) in those who are at risk of progressing to heart failure, it may help slow down progression by more aggressive management of the problem.

Though there is fair body of evidence on the mechanism underlying the decline in exercise tolerance and despite the recognition of its prognostic significance, monitoring microvascular changes underlying altered skeletal muscle exercise capacity and mapping them dynamically against cardiac response capacity has not been attempted. Monitoring of regional alterations in the microcirculation in patients is restricted by the invasive character of methodology and limitations to its adaptation to record continuous and dynamic change in the exercising muscle. Assessing cardiac function during exercise poses a special challenge as conventional echocardiographic measures including strain analysis, can only be done at rest or at the end of exercise. Therefore dynamic response to exercise, which is a function of continuous adjustment of pre-load (Frank-Starling) and after-load as well as direct cardiac adrenergic influence, designed to optimise the inotropic and chronotropic responses, cannot be captured by these methods.

Impedance cardiography (ICG) has been validated to gather ambulatory physiological recordings and was adapted to monitor cardiac function online during exercise. ${ }^{12}$ For monitoring regional microcirculation this was combined with another method where laser Doppler shift caused by the movement of erythrocytes in the detected laser light is used to determine arteriolar flow. With the same probe, white light is introduced into the tissue and oxygen saturation of haemoglobin was determined from light absorbed by the haemoglobin in mixed capillary venous blood at a predetermined depth from the skin. ${ }^{3}$ This study was therefore aimed at investigating the sequential changes during mild exercise and recovery in cardiac function and regional microcirculation of exercising muscle in diabetes by a method which allows continuous dynamic monitoring, in order to capture early cardiac and vascular correlates, which could ultimately progress to impaired exercise tolerance. Submaximal exercise is more relevant as it is equivalent to the 6-minute walk test, used to evaluate exercise capacity in patients with marked left ventricular (LV) dysfunction. ${ }^{4}$ In contrast, maximum oxygen consumption $\left(\mathrm{VO}_{2} \mathrm{max}\right)$ may be compromised because of reduced patient motivation, poor peripheral blood flow and impaired skeletal muscle metabolism with early development of acidosis, besides limitation in cardiac output, correlates only modestly with this test. ${ }^{5}$

\section{STUDY PROTOCOL}

\section{Subjects}

The investigation conforms to the principles outlined in the Declaration of Helsinki. ${ }^{6}$ The study was approved by the institutional review board of Torrent Research Centre and Veeda Clinical Research (Ahmedabad, India). After signing the written informed consent, 127 subjects were screened. The inclusion criteria were subjects with 
confirmed diabetes as ascertained by history, clinical examination and laboratory investigations, who had no history of breathlessness on exertion or limitation in exercise capacity, and age-matched controls who were non-diabetic. Subjects with arrhythmia, significant peripheral vascular disease, retinopathy, insulin dependence and those with blood pressure (BP) $>180$ / $100 \mathrm{~mm} \mathrm{Hg}$, were excluded from the study. One-hundred and one subjects were included in the study and all completed the exercise protocol. Data from diabetics with glycosylated haemoglobin $\left(\mathrm{HbA}_{1 \mathrm{c}}\right)<8(\mathrm{n}=31)$, diabetics with $\mathrm{HbA}_{1 \mathrm{c}} \geqslant 8$ $(n=38)$ and age-matched normal controls $(n=32)$ were included in the analysis.

\section{Experimental procedure}

At Veeda Clinical Research, after their breakfast subjects had a brief physical examination, blood samples were collected for estimation of $\mathrm{HbA}_{1 \mathrm{c}}$, vascular cell adhesion molecule (VCAM), $\mathrm{N}$-terminal brain natriuretic propeptide (NTProBNP) and urine samples for creatinine and microalbumin estimation. Oxygen to See (O2C, LEA Medizintechnik, Germany) probe LF3 incorporating Doppler (830 nm) and near infra-red light spectrum (500$850 \mathrm{~nm}$ ), which allows measurement at skeletal muscle postcapillary venular site, was placed on the belly of the gastrocnemious on one leg. ICG (Bioz, Cardiodynamics, USA) and electrocardiogram (ECG-Mac5000, GE Healthcare, Germany) probes were placed on the chest and BP cuff on the arm.

After calibration, continuous measurements were obtained at baseline in the resting supine position for 10 minutes followed by the upright position for 10 minutes and then acclimatisation on the bicycle ergometer (eBike, GE Healthcare, Germany) for 10 minutes. Exercise was for a duration of 10 minutes, at a constant load of $50 \mathrm{~W}$, on the electrically braked ergometer, varying rpm to maintain the constant load, which is equivalent to 4-7 METS. This level of exercise was chosen to simulate a 6minute walk test commonly used to evaluate patients with heart failure in order to determine if it was adequate to evoke peripheral vascular response to differentiate the diabetic from non-diabetic subjects. At the end of the exercise period, subjects were asked to lie supine again for a recovery period of at least 15 minutes. ECG was recorded throughout and BP every 2 minutes. The same protocol was repeated in 10 subjects to assess the repeatability of the measurements. The entire study was completed over a period of four months.

\section{Data acquisition and processing}

Data from O2C and ICG were acquired for five different phases-namely, resting supine baseline, standing, sitting on ergometer, during exercise and recovery after exercise. ICG measurements (collected every 1 minute) and $\mathrm{O} 2 \mathrm{C}$ measurements (collected every 2 seconds were also averaged for 1 minute) were classified and synchronised for different phases of the protocol. Data were cleaned for machine error and artefacts (random and during transition from one phase of protocol to next) for all the measured variables. For O2C parameters, flow response to exercise was measured as area under the curve (AUC) and oxygen saturation $\left(\mathrm{SO}_{2}\right)$ as area over the curve (AOC) of percentage change at each time point during exercise, from a mean of sitting. In addition, the slope to achieve maximum velocity in the microcirculation was computed. ICG parameters considered for analysis were cardiac output (CO), stroke volume (SV) and systolic time interval. Systolic time interval was obtained by summing the pre-ejection period (PEP) and left ventricular ejection time (LVET) obtained from ICG.
Response during recovery was measured as AUC of the data points recorded during the recovery phase. Subjects who completed the exercise phase of 10 minutes were included for analysis on exercise data (table 1). For analysis on recovery data, only those who completed exercise followed by 15 minutes of recovery were included (28 diabetics with $\mathrm{HbA}_{1 \mathrm{c}}<8,35$ diabetics with $\mathrm{HbA}_{1 \mathrm{c}} \geqslant 8$ and 32 age-matched normal controls). To calculate oxygen debt for each subject, the value of mean $\mathrm{SO}_{2}$ at resting supine baseline, multiplied by the same period of time-that is, 15 minutes, was subtracted from AUC over 15 minutes of recovery. ${ }^{7}$ AUC has been calculated using WinNonlin (Version-5.2).

\section{Statistical analysis}

Data are expressed as mean (SD) and graphically represented as mean (SEM). Statistical analysis has been performed using SAS (Version-9.1). Demographic data have been tested to see differences among three groups using ANOVA followed by post-hoc Dunnett $t$ tests (two-sided). Repeatability was determined by a two-sided paired t test on AUC values (derived from percentage change during exercise from sitting baseline) of the $\mathrm{O} 2 \mathrm{C}$ parameters, $\mathrm{SO}_{2}$ and flow and ICG parameter, $\mathrm{CO}$. Proportions of concomitant medications were subjected to Fisher's exact test. CO, SV, time for downward inflection, and AUC or AOC of percentage change from baseline, during exercise and for recovery period, was compared between groups by the one-sided unpaired t test. In the absence of normality, data were subjected to the non-parametric Wilcoxon test. Percentage change in systolic time interval over time, of normal, diabetics with $\mathrm{HbA}_{1 \mathrm{c}}<8$ and diabetics with $\mathrm{HbA}_{1 \mathrm{c}} \geqslant 8$, were compared by repeated measures analysis of variance (RMANOVA). Statistical significance was defined as $p$ value $\leqslant 0.05$.

\section{RESULTS}

\section{Demography}

The demographic profile of the subjects included in the study is shown in table 1. Medications, sulphonylurea secretagogues, thiazolidinedione sensitisers, antihypertensives, angiotensinconverting enzyme (ACE) inhibitors and angiotensin receptor blockers (ARBs) and loop diuretics, as well as statins and $\beta$ blockers (table 1), taken by the subjects did not influence the measurements made during the study. Statins were analysed separately and did not show an effect on the cardiac response to exercise when comparing the two diabetic groups. Similarly, the diabetic group with $\mathrm{HbA}_{1 \mathrm{c}} \geqslant 8$, showed a greater attenuation of the cardiac response to exercise as compared to the diabetic group with $\mathrm{HbA}_{1 \mathrm{c}}<8$. This was probably not secondary to attenuation of the response due to $\beta$-blockers as fewer diabetics with $\mathrm{HbA}_{1 \mathrm{c}} \geqslant 8$ were taking $\beta$-blockers compared to those with $\mathrm{HbA}_{1 \mathrm{c}}<8$.

\section{Cardiac response during exercise}

Resting CO was lowest in normal individuals and although statistically not significant, it was slightly higher in the diabetic groups with $\mathrm{HbA}_{1 \mathrm{c}}<8$ and $\geqslant 8$. In response to exercise, there was a rise in both SV and $\mathrm{CO}$ in all groups. However, the capacity to maximally increase $\mathrm{CO}$ in response to exercise, a measure of the cardiac reserve, was significantly $(p<0.001)$ reduced in the diabetic patients compared to normal subjects (fig 1A). This could be ascribed to a smaller rise in SV during exercise in diabetic patients, which was significantly reduced $(p<0.002)$ in the more severely diabetic group (fig 1B). 
Table 1 Demographic profile of subjects

\begin{tabular}{|c|c|c|c|}
\hline & Normal $(n=32)$ & $\mathrm{HbA}_{1 \mathrm{c}}<8(\mathrm{n}=31)$ & $\mathrm{HbA}_{1 \mathrm{C}} \geqslant 8 \quad(\mathrm{n}=38)$ \\
\hline Age (years) & $48.8(5.3)$ & $50.6(7.3)$ & $50.3(4.6)$ \\
\hline $\mathrm{BMI}\left(\mathrm{kg} / \mathrm{m}^{2}\right) \ddagger$ & $23.81^{*}(3.4)$ & $26.42(2.8)$ & $26.57(3.8)$ \\
\hline Systolic blood pressure (mm Hg) & $116(10)$ & $120(12)$ & $117(11)$ \\
\hline Diastolic blood pressure $(\mathrm{mm} \mathrm{Hg})$ & $80(9)$ & $80(7)$ & $78(9)$ \\
\hline Cardiac output (I/ml) & $4.96(0.95)$ & $5.10(0.91)$ & $5.19(0.76)$ \\
\hline Heart rate & $77(11)$ & $80(14)$ & $80(10)$ \\
\hline NT-proBNP $(\mathrm{pg} / \mathrm{mll}) \dagger$ & $31(14.5-51.5)$ & $35.1(11.5-62.7)$ & $27.6(14.6-55.7)$ \\
\hline $\operatorname{VCAM}(\mathrm{ng} / \mathrm{ml}) \dagger$ & $326.8(186-647)$ & 343.8 (171-617) & $416.9(253-611)$ \\
\hline Diabetes duration (years) $\dagger$ & - & $4(3-10)$ & $5(3-10)$ \\
\hline Microalbuminuria (mg/l) & $18.99(44.93)$ & $17.85(36.35)$ & $41.05(88.11)$ \\
\hline $\operatorname{eGFR}\left(\mathrm{ml} / \mathrm{min} / 1.73 \mathrm{~m}^{2}\right)$ & $85(12)$ & $80(16)$ & $90(17)$ \\
\hline \multicolumn{4}{|l|}{ Concomitant medication } \\
\hline Secretagogues & - & 70 & 73 \\
\hline Sensitisers & - & 73 & 76 \\
\hline$\beta$-blockers: & - & 33 & $5^{* *}$ \\
\hline Antihypertensives & - & 50 & 34 \\
\hline Diuretics & - & 7 & 3 \\
\hline Statins & _ & 8.3 & 0 \\
\hline
\end{tabular}

Values are means (SD) or tmedian (interquartile range).

${ }^{*} p<0.01$ normal vs $<8$, normal vs $\geqslant 8 ;{ }^{* *} p<0.05<8$ vs $\geqslant 8$.

$\$$ Does not alter and contribute to diabetic vs non-diabetic comparison for various ICG and $02 \mathrm{C}$ variables.

Following the initial rise, the time to downward inflection towards reduction of SV, an indicator of inadequate filling secondary to increasing heart rate, occurred significantly $(p<0.05$ normal vs $<8$; $p<0.001$ normal vs $\geqslant 8$ ) earlier, and at lower heart rates ( $p<0.05$ normal vs diabetic groups) in the diabetic groups compared to normal (fig $2 \mathrm{~A}$ and $2 \mathrm{~B}$ ). Lengthening of the systolic time interval was observed in the diabetic group with $\mathrm{HbA}_{1 \mathrm{c}} \geqslant 8$, in that there was a greater compensatory lengthening of the systolic time interval depicted here as a smaller fall in the systolic time for a given stroke output during exercise compared to normal $(\mathrm{p}<0.05)$ (fig 2C).

\section{Cardiac response during recovery}

During the recovery phase after exercise, the $\mathrm{CO}$ remained elevated and did not return to resting baseline. The increase however was attenuated in diabetics, more so in the diabetics with $\mathrm{HbA}_{1 \mathrm{c}} \geqslant 8(\mathrm{p}<0.05)$ (fig $\left.3 \mathrm{~A}\right)$. There was no difference in the heart rate response during recovery between the different groups.

\section{Skeletal muscle perfusion during exercise}

Regional changes in perfusion in the muscle as revealed by local measurement of flow are shown in figure 4A. There was a significant $(p<0.05)$ reduction in AUC of percentage change from baseline in diabetic individuals with $\mathrm{HbA}_{1 \mathrm{c}} \geqslant 8$ (1024.7 (780.3)) compared to normal subjects (1433.4 (1055.9)). Diabetics with $\mathrm{HbA}_{1 \mathrm{c}}<8$, on the other hand, did not show a significant difference; however, to compensate, flow was slightly increased (1541.1 (1648.1)) compared to normal (fig 4A). This was accompanied by a reduction in oxygen saturation in both the diabetic groups compared to normal (fig 4B). AOC in normal subjects (-77.2 (170.8)) was significantly $(\mathrm{p}<0.05)$ more compared to diabetics with $\mathrm{HbA}_{1 \mathrm{c}}$ $<8(-125.3(236.1))$ and diabetics with $\mathrm{HbA}_{1 \mathrm{c}} \geqslant 8(-165.6$ (247.6)). This was offset, in the diabetics with $\mathrm{HbA}_{1 \mathrm{c}} \geqslant 8$, by increased velocity of blood in the microcirculation, which showed a significant increase $(p<0.05)$ in the slope to achieve maximal velocity (slope $=3.05(2.59)$ ), compared to the normal subjects (slope $=2.08(1.65)$ ).
Figure 1 (A) Cardiac reserve during exercise and $(B)$ stroke volume reserve during exercise, the key parameter limiting increase in cardiac output. $\mathrm{CO}$, cardiac output; $\mathrm{HbA}_{1 \mathrm{c}}$, glycosylated haemoglobin.
A

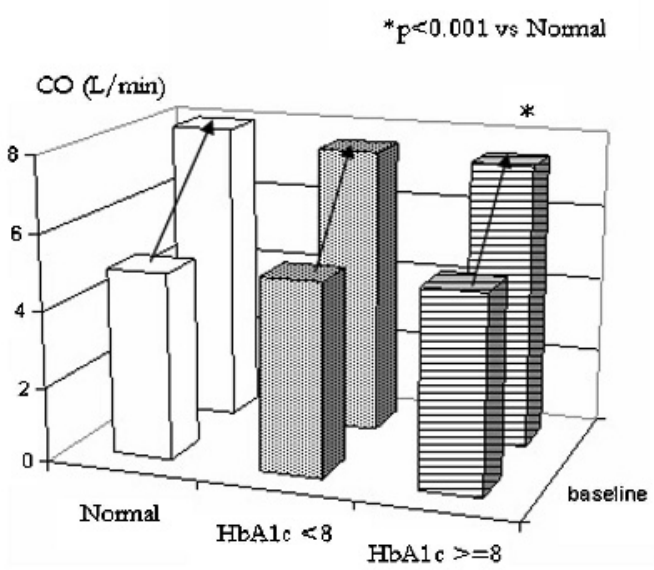

B

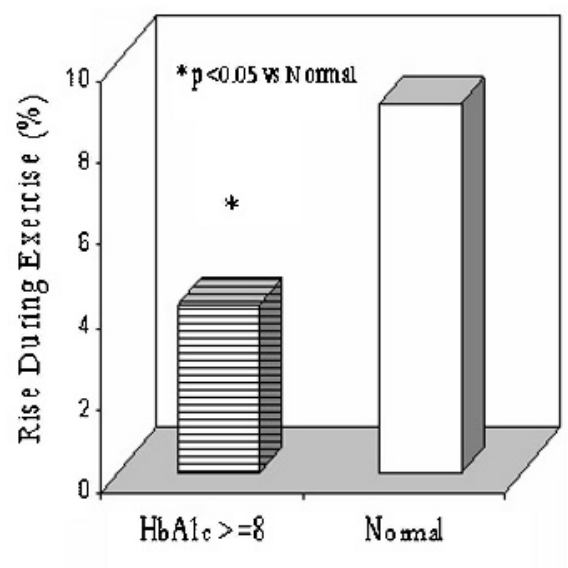


Figure 2 (A) Time to downward inflection of stroke volume response during exercise demonstrating that with increasingly poor glycaemic control there is increasing limitation in the capacity to augment stroke volume. (B) Heart rate at downward inflection in stroke volume during exercise. Increase in cardiac output occurs as a function of increase in heart rate with decreasing oxygen pulse delivered to muscles. (C) Reduction in systolic time interval compared to baseline indicating longer systole and compromised diastole in diabetics. $\mathrm{HbA}_{1 \mathrm{c}}$, glycosylated haemoglobin; RMANOVA, repeated measures analysis of variance.
A

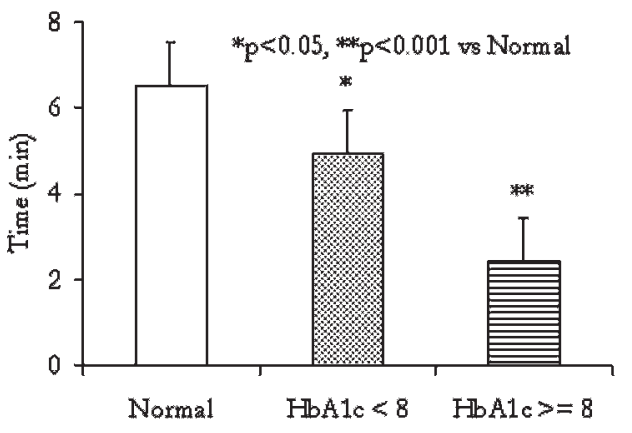

C

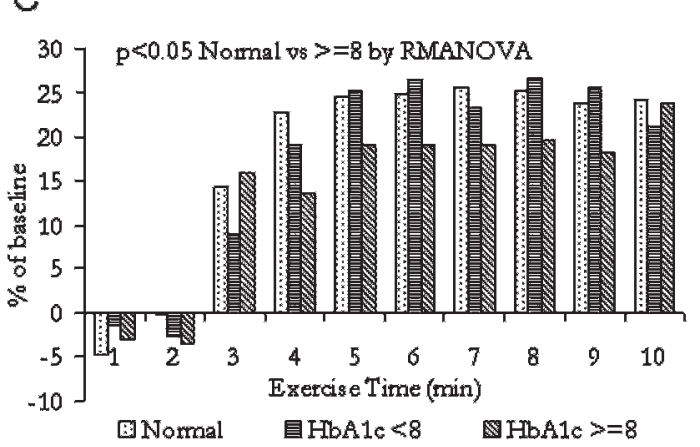

B

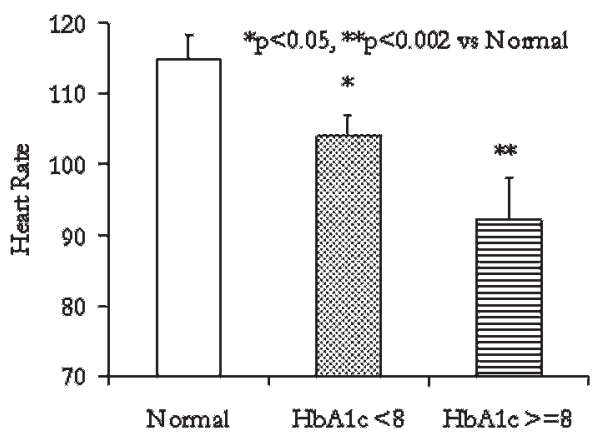

\section{Skeletal muscle perfusion during recovery}

Regional flow to the exercising muscle normally remains elevated, over resting levels, even after stopping exercise, during recovery. Compared to normal subjects, in diabetics augmentation of flow was more (fig $3 \mathrm{~B}$ ), in order to compensate for the reduced $\mathrm{CO}$ response during recovery.

Oxygen debt as determined from the diminished $\mathrm{SO}_{2}$ during recovery, over the resting baseline, was significantly increased in the diabetic subjects compared to normal (fig $3 C)(p<0.05)$. Despite the increase in flow, desaturation of the local flow over the resting values persisted; however, it was least in the normal subjects, as evident by highest AUC of oxygen saturation during recovery. In the diabetic group, $\mathrm{SO}_{2}$ reduced significantly in both mild and severely diabetic patients (fig 3D).

\section{Repeatability}

Data from 10 subjects who repeated the exercise protocol showed that AUC of percentage change from the sitting position to exercise did not show any significant difference in $\mathrm{SO}_{2}(507$ (158) initial vs 625 (126) repeat, $\mathrm{p}>0.05)$, flow (3400 (867) initial vs 3166 (996) repeat, $\mathrm{p}>0.05)$ and in CO (75.6 (13.3) initial vs 75.7 (13.8) repeat, $p>0.05)$. Hence, the precision of the relative measurements before and during exercise were reasonably accurate for acceptance of O2C and ICG.
Figure 3 (A) Difference in area under the curve (AUC) of cardiac output (CO) during recovery from resting baseline: cardiac output overshoot during recovery. (B) Increase in regional flow over baseline during recovery: difference in AUC during recovery from resting baseline. (C) Oxygen debt: difference in AUC during recovery from resting baseline. (D) Oxygen saturation (AUC) in muscle flow during recovery. $\mathrm{HbA}_{1 \mathrm{c}}$, glycosylated haemoglobin.
A

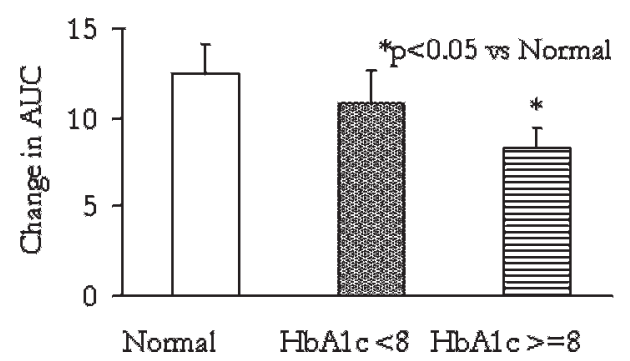

C

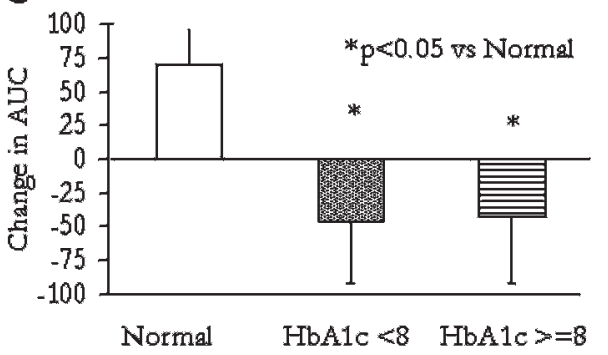

B

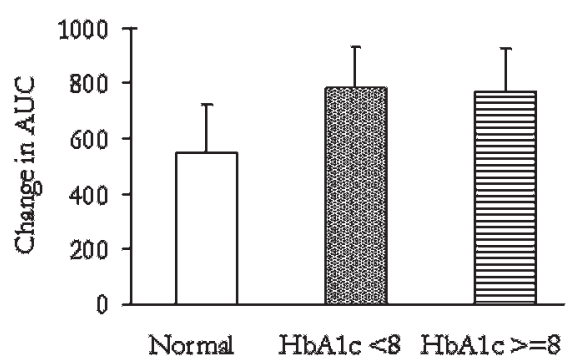

D

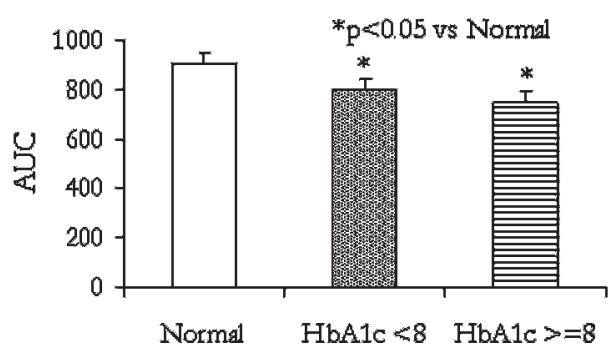


Figure 4 (A) Regional muscle flow during exercise and (B) oxygen saturation in blood supplied to exercising muscle. Note that in diabetics with reasonable control increase in flow is not able to contain desaturation. Diabetics with poor control show limited capacity to augment flow and run up a steeper oxygen debt with a late attempt at correction which could be due to local factors over-riding systemic vascular tone. $\mathrm{HbA}_{1 \mathrm{c}}$ glycosylated haemoglobin.
A

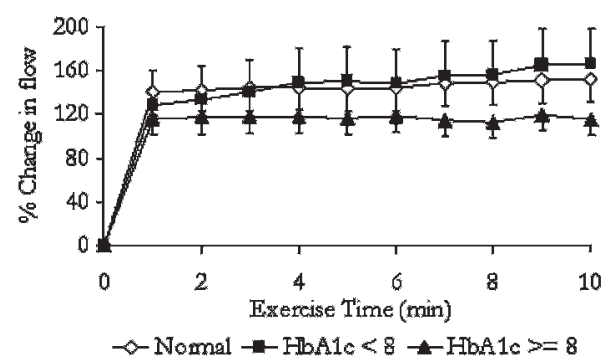

B

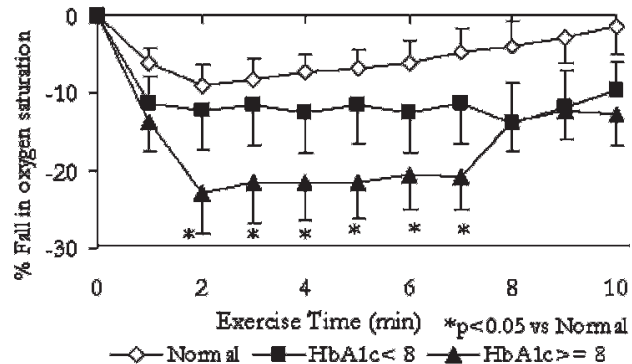

\section{DISCUSSION}

This study characterises the haemodynamic response to exercise in diabetic individuals compared to normal individuals, by dynamic recording of cardiac function as well as simultaneous monitoring of regional perfusion of the muscle. These findings demonstrate that cardiac response even to submaximal exercise is attenuated significantly in diabetic individuals. Simultaneously, there is an attempt to maintain regional microvascular perfusion by a compensatory augmentation of regional flow to the exercising muscle. Despite this compensation, there is greater desaturation of the supply reaching the working muscle in diabetics compared with normal individuals. Furthermore, the reduction in cardiac reserve as well as regional oxygen delivery during exercise, were found to be related to the severity of diabetes.

Reduced exercise capacity in diabetics has been reported in subjects with non-insulin dependent diabetes mellitus (NIDDM) without coronary artery disease ${ }^{8}$ and a correlation between left ventricular dysfunction and impaired exercise capacity has been demonstrated in diabetics in several studies. ${ }^{9}$ Our study confirms that the SV reserve is the key parameter that is compromised during exercise in asymptomatic diabetic individuals. This is evident even with the mild exercise equivalent to a 6-minute walk test when measured dynamically in ambulatory subjects who are not in failure. The association of poor glycaemic control with worse cardiac and peripheral dysfunction in this study is consistent with a previous large study in asymptomatic type 2 diabetics. ${ }^{10}$ Other studies have shown that $A_{1 c}$ has an inverse correlation with maximum oxygen uptake, ${ }^{11}$ work capacity ${ }^{12}$ and exercise duration. ${ }^{13}$

Perturbation of cardiac function independent of coronary artery disease and BP in diabetes may result from an interplay of a wide variety of progressively dysfunctional autoregulatory mechanisms. Diastolic abnormalities like delayed onset of ventricular relaxation, ${ }^{14}$ and systolic function abnormalities of the ventricular muscular pump characterised by abnormal systolic time intervals and by increased brain natriuretic peptide (BNP) levels, as observed in this study, precede reduced haemodynamic pump performance with severe systolic and diastolic abnormalities. Limitations on cardiac reserve and cardiac contractile function thus restrict the supply from meeting the demand imposed by exercise. In addition, microcirculatory deficits coupled with altered energetics limit the ability to meet the demand in exercising skeletal muscle in diabetics. ${ }^{15}{ }^{16}$ Endothelial dysfunction and functional alterations of the microcirculation, on the other hand, precede morphological changes and contribute to an increased vascular permeability $^{17}$ and an impaired endothelial vasodilatory response. There are several reports that insulin also increases sympathetic nervous system activity, which can determine the nutritive flow distribution. ${ }^{18}$ Diabetes with associated insulin resistance and autonomic dysfunction could lead to further exacerbation of the maldistribution locally to fulfil the nutritive flow required to meet the metabolic demand during exercise. These changes, acting in conjunction, induce dysfunction with resultant reductions in flow and $\mathrm{O}_{2}$ delivery at rest and during exercise. ${ }^{19}$ A perturbation of intramuscular homeostasis in response to exercise challenge was also observed in this study, which has the potential to contribute to premature muscular fatigue and progress to reduced exercise tolerance in diabetic individuals.

During the recovery period, a shift from carbohydrate to fat as a fuel substrate occurs to prevent further depletion of muscle glycogen stores, leading to oxygen consumption remaining elevated above resting levels after exercise, called oxygen debt, because the oxygen cost of fat catabolism is greater than that of carbohydrate catabolism. ${ }^{20}{ }^{21}$ In order to meet this requirement, a marked increase in stroke volume during early recovery with overshoot appears to be facilitated by both an immediate afterload reduction and a relatively slow decrease in cardiac sympathetic stimulation. Accumulation of a large oxygen debt during exercise has been reported in heart failure patients. ${ }^{22}$ In this study, overshoot of cardiac output was observed during recovery, though lower in diabetics and so was the mixed venous $\mathrm{SO}_{2}$, suggesting more severe muscle underperfusion and greater $\mathrm{O}_{2}$-deficit and regional ischaemia in diabetic individuals. A possible mechanism could be a persistence of sympathetic activity, which serves to increase vascular resistance in nonworking tissues during exercise, which the local metabolic factors and endothelium-derived substances such as nitric oxide and prostaglandins are unable to over-ride, as they are deficient in the diabetic state. Increased renin-angiotensin-endothelin system activity may also contribute, at least in part.

Mediators of the changes underlying impaired exercise response in patients with type 2 diabetes are multiple. Limited exercise tolerance has been associated with poor glycaemic control. $^{8}{ }^{10-1223}$ Secondary to this, glycosylation leading to the formation of AGE (advanced glycation endproduct), initiate a proinflammatory and procoagulant state and may impair the function of a number of proteins leading to cardiac, vascular or endothelial dysfunction. ${ }^{24} 25$ We have found that diabetesinduced myocardial dysfunction in a diabetic hypertensive rat model was reversed by reducing the AGE burden consequent to treatment with AGE-breaker. ${ }^{26}$

Previous reports of ICG in diabetics have been limited and restricted to measurements at rest. One ICG study in diabetic individuals revealed that thoracic fluid content increased despite improvement in contractile indices after treatment with rosiglitazone. ${ }^{27}$ In another study, no difference was detected in cardiac index at rest between healthy individuals and newly diagnosed diabetics ${ }^{28}$ indicating that possibly exercise provocation would be 
necessary to bring out the subtle differences in the haemodynamics. Insights on the control of muscle blood flow and oxygen consumption during exercise have, in the past, relied on measurements of bulk flow in large conduit arteries to the exercising limbs precluding analysis of local perfusion and oxygenation mismatch. However, comparing changes during rest, dynamic exercise and recovery after exercise with a combination of NIRS, laser Doppler and impedance cardiography to expose subclinical differences in diabetic individuals has not been attempted previously to the best of our knowledge. Repeatability of the measurements showed reasonable consistency between measurements made on both $\mathrm{O} 2 \mathrm{C}$ and ICG parameters.

The potential limitations of our study are the mixed diabetic population studied, which could have incipient complications like neuropathy, peripheral vascular disease and coronary artery disease, which could be influencing the study parameters. For diabetic subjects without any signs of heart failure, reduction in exercise capacity secondary to reduction in cardiac output may be evident only during maximal exercise and could be of prognostic significance. We also do not have tissue-Doppler data to correlate limitation in increasing stroke volume to diastolic dysfunction.

\section{CONCLUSION}

In summary, this cross-sectional study in a diabetic population showed that by combining the assessment of dynamic cardiac response during exercise with peripheral flow distribution and nutritive perfusion, subclinical differences in haemodynamic response were detected in diabetic individuals, hitherto not reported to the best of our knowledge. Longitudinal follow-up of these patients and a comparison of dynamic cardiac response and that of peripheral flow and nutritive distribution, as described, with the traditional measures of exercise capacity, which have been studied longitudinally over time and shown to have prognostic significance, would provide an objective and sensitive measure of cardiac and microvascular correlates of the composite haemodynamic function and indicate the suitability of such diagnostic measures for early detection of a population at risk of progressing to heart failure. ${ }^{5}$

Acknowledgements: We are grateful to Dr Jinesh Shah, Mr Anand Chettiyar and $\mathrm{Mr}$ Nilesh Badgujar for assistance in conduct and data management of the study and Ms Shikha Kumar for editorial assistance.

Funding: The study was funded by Torrent Pharmaceutical Limited.

Competing interests: DJ, AS, AV and CD are employees of Torrent Pharmaceutical Limited, Ahmedabad, and MRS and SKS are employees of Veeda UK and Veeda India, respectively.

Work was carried out at Discovery Biology, Torrent Research Centre, Village Bhat, Gandhinagar 382428, Gujarat, India and Clinical Pharmacology Unit, Veeda Clinical Research, Shivalik Plaza-A, Near IIM, Ambawadi Ahmedabad 380015, Gujarat, India.

Provenance and peer review: Not commissioned; externally peer reviewed.

\section{REFERENCES}

1. Thomas SH, Crowther A. Impedance cardiography during exercise in patients with coronary heart disease. Eur Heart J 1993;14:150-9.
2. Greenberg BH, Hermann DD, Pranulis MF, et al. Reproducibility of impedance cardiography hemodynamic measures in clinically stable heart failure patients. Congest Heart Fail 2000;6:74-80.

3. Beckert S, Witte MB, Koenigsrainer A, et al. The impact of the micro-lightguide O2C for the quantification of tissue ischemia in diabetic foot ulcers. Diabetes Care 2004;27:2863-7.

4. Bittner V, Weiner DH, Yusuf S, et al. Prediction of mortality and morbidity with a 6minute walk test in patients with left ventricular dysfunction: SOLVD Investigators. JAMA 1993;270:1702-7.

5. Davies LC, Wensel R, Georgiadou P, et al. Enhanced prognostic value from cardiopulmonary exercise testing in chronic heart failure by non-linear analysis: oxygen uptake efficiency slope. Eur Heart J 2006;27:684-90.

6. Rickham PP. Human Experimentation code of ethics of the world medical association. Declaration of Helsinki. BMJ 1964;ii:177.

7. Aliverti A, Dellacà RL, Lotti $P$, et al. Influence of expiratory flow-limitation during exercise on systemic oxygen delivery in humans. Eur J Appl Physiol 2005;95:229-42.

8. Estacio RO, Regensteiner JG, Wolfel EE, et al. The association between diabetic complications and exercise capacity in NIDDM patients. Diabetes Care 1998;21:291-5

9. Fang ZY, Sharman J, Prins JB, et al. Determinants of exercise capacity in patients with type 2 diabetes. Diabetes Care 2005;28:1643-8.

10. Demir I, Ermis C, Altunbas $\mathrm{H}$, et al. Serum $\mathrm{HbA1c}$ levels and exercise capacity in diabetic patients. Jpn Heart J 2001;42:607-16.

11. Vanninen $\mathbf{E}$, Uusitupa $\mathrm{M}$, Siitonen 0 , et al. Habitual physical activity, aerobic capacity and metabolic control in patients with newly-diagnosed type 2 (non-insulindependent) diabetes mellitus: effect of 1-year diet and exercise intervention. Diabetologia 1992:35:340-6.

12. Barkai L, Peja M, Vamosi I. Physical work capacity in diabetic children and adolescents with and without cardiovascular autonomic dysfunction. Diabet Med 1996;13:254-8.

13. Baum VC, Levitsky LL, Englander RM. Abnormal cardiac function after exercise in insulin-dependent diabetic children and adolescents. Diabetes Care 1987;10:319-23.

14. De Keulenaer GW, Brutsaert DL. Systolic and diastolic heart failure: different phenotypes of the same disease? Eur J Heart Fail 2007:9:136-43.

15. Scheuermann-Freestone M, Madsen PL, Manners D, et al. Abnormal cardiac and skeletal muscle energy metabolism in patients with type 2 diabetes. Circulation 2003;107:3040-6.

16. Wu YW, Hsu CL, Wang SS, et al. Impaired exercise capacity in diabetic patients after coronary bypass surgery: effects of diastolic and endothelial function. Cardiology 2008;110:191-8.

17. Kingwell BA, Bradley SJ, Formosa BA, et al. Type 2 diabetic individuals have impaired leg blood flow responses to exercise: role of endothelium-dependent vasodilation. Diabetes Care 2003;26:899-904.

18. Vollenweider $\mathbf{P}$, Randin D, Tappy L, et al. Impaired insulin-induced sympathetic neural activation and vasodilation in skeletal muscle in obese humans. J Clin Invest 1994;93:2365-71.

19. Creager MA, Luescher TF, Cosentino F, et al. Diabetes and vascular disease. Pathophysiology, clinical consequences, and medical therapy: Part I. Circulation 2003;108:1527-32

20. Wong T, Harber V. Lower excess postexercise oxygen consumption and altered growth hormone and cortisol responses to exercise in obese men. J Clin Endocrinol Metab 2006;91:678-86.

21. Kano H, Koike A, Yajima T, et al. Mechanism of overshoot in cardiac function during recovery from submaximal exercise in man. Chest 1999;116:868-73.

22. Mitchell SH, Steele NP, Leclerc KM, et al. Oxygen cost of exercise is increased in heart failure after accounting for recovery costs. Chest 2003;124:572-79.

23. Fang ZY, Prins JB, Marwick TH. Diabetic cardiomyopathy: evidence, mechanisms, and therapeutic implications. Endocr Rev 2004;25:543-67.

24. Wautier JL, Schmidt AM. Protein glycation: A firm link to endothelial cell dysfunction. Circ Res 2004;95:233-8.

25. Kelley DE, He J, Menshikova EV, et al. Dysfunction of mitochondria in human skeletal muscle in type 2 diabetes. Diabetes 2002;51:2944-50.

26. Pathak P, Gupta R, Chaudhari A, et al. TRC4149 a novel advanced glycation end product breaker improves hemodynamics status in diabetic spontaneously hypertensive rats. Eur J Med Res 2008;13:388-98.

27. Ferdowsali K, Tadlaoui K, Nguyen E, et al. Rosiglitazone induced changes in the hemodynamic profile of patients assessed by impedance cardiography. Circulation 2006;114:II 881 Abstract.

28. Loimaala $\overline{\mathbf{A}}$, Groundstroem K, Majahalme S, et al. Impaired myocardial function in newly onset type 2 diabetes associates with arterial stiffness. Eur J Echocardiogr 2006; 7:341-7. 